\title{
A new leafhopper genus of Erythroneurini (Hemiptera, Cicadellidae, Typhlocybinae) from karst area in southwestern China
}

\author{
Ni Zhang ${ }^{1}$ (D), Qing-fa Songำ, Yue-hua Song ${ }^{1 *}$ \\ ${ }^{1}$ Guizhou Normal University, School of Karst Science, State Key Laboratory Cultivation Base for Guizhou Karst Mountain Ecology \\ Environment of China, Guiyang, China. \\ urn:1sid:zoobank.org:pub:2FA99EFA-9647-498D-B341-19BE0C7C4E9E
}

\section{A R T I C L E I N F O}

\section{Article history:}

Received 14 April 2021

Accepted 06 December 2021

Available online 14 January 2022

Associate Editor: Daniela Takiya

\section{Keywords:}

Hemiptera

Auchenorrhyncha

Erythroneurini

Taxonomy

New taxa

Karst

\begin{abstract}
A B S T R A C T
Guizhou is regarded as one hotspot for China's Erythroneurini diversity, from where numerous rare new species have been, or are being, described. A new genus and species of Erythroneurini from Huajiang, Guizhou, China, Anuihuajianga pyramidalis gen. et sp. nov. are described and illustrated. The new genus is characterized by subgenital plate extremely short, with long macrosetae dorsally at base, and small teeth-like setae at apex. The characteristic aedeagus shaped like two overlapping cones can also easily distinguish it from other genera. In general, this study further enriches the diversity of Erythroneurini in southwestern China.
\end{abstract}

\section{Introduction}

Erythroneurini Young, 1952 is the largest leafhopper tribe of Typhlocybinae (Young, 1952). The subfamily comprises 204 genera and 2,000 described species (Dmitriev, 2003; Song et al., 2016; Song and $\mathrm{Li}, 2017$ ), and is widely distributed in the six major zoogeographic regions of the world (Chen et al., 2020). They are important agricultural and forestry pests that feed on an extensive variety of plants, including cereal crops, vegetables, fruit trees and wild grasses, and they are also vectors of plant disease through a combination of herbivory and pathogen transmission (Morris, 1971; Guo, 2011; Roddee et al., 2018). Host plants for most species remain unknown, but species with known hosts appear to be highly host-specific (Jiang et al., 2005; Jarrell et al., 2020; Kim et al., 2021).

Guizhou is located at the eastern slope of the Yunnan-Guizhou Plateau in southwestern China, which is suitable for long-term inhabitation of leafhoppers in view of abundant rainfall, suitable temperature, and high vegetation coverage. A total of 28 genera and 113 species of

\footnotetext{
*Corresponding author:

E-mail: songyuehua@163.com (Y. H. Song).
}

Erythroneurini have been recorded in Guizhou, accounting for $54 \%$ and $33 \%$ of the recorded genera and species in China by 2020 (Chen et al., 2020). However, many genera and species from this area remain to be described, thus there is an urgent need to collect and describe the diversity of leafhoppers. The objective of this work was to describe a new genus and species from southwestern China.

\section{Material and methods}

All leafhoppers were collected and preserved in absolute ethanol. Morphological terminology used in this work follows Dietrich (2005) and Song and $\mathrm{Li}$ (2013). Abdomens of specimens were removed and cleared in hot $10 \% \mathrm{NaOH}$, rinsed with water, and then stored in glycerin. Genital dissections were performed in glycerin to prevent pieces from drying out. The dissected male genitalia were observed and drawn using Olympus SZX16 and BX53 microscopes. Images of habitus were taken using a KEYENCE VHX-5000 digital microscope. Body length was measured from apex of vertex to tip of forewings. Multiple images were stacked to obtain focused pictures and edited in Adobe Photoshop CS6. 
All specimens examined are deposited in the collection of the School of Karst Science, Guizhou Normal University, China (GZNU).

\section{Results}

\section{Anuihuajianga Zhang \& Song gen. nov.}

urn:Isid:zoobank.org:act: BFF7904F-7D20-4B8E-A361-C737087595EB (Figs. 1-3)

\section{Type species. Anuihuajianga pyramidalis Zhang \& Song sp. nov.}

Diagnosis. Anuihuajiangagen. nov. can be distinguished from other genera of Erythroneurini by the following combination of characters: 1) fourth apical cell of forewing very small and triangular; 2) subgenital plate short and stubby, with several macrosetae in two groups, and peg-like setae on preapical projection; 3) connective M-shaped, with central lobe; 4) aedeagus with shaft short, slightly "S" sinuated; and 5) anal tube with segment $X$ with paired basal processes and paired bifurcated ventroapical processes.

Description. Whole body greenish grey. Head, in dorsal view, roundly produced; narrower than pronotum; its medium length much shorter than width between eyes; with paired preapical dark spots. Pronotum nearly twice as long as vertex; almost transparent. Face broad; distinctly broadened at lower part; convex in profile. Forewing semitransparent; with four apical cells, $1^{\text {st }}$ apical cell with angulate

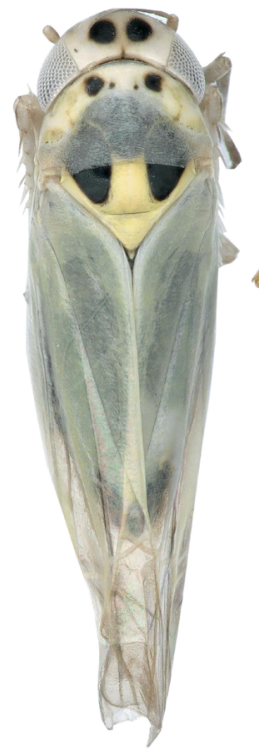

A

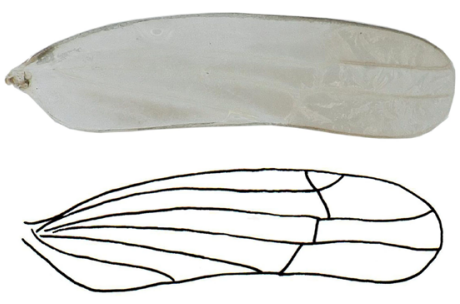

$\mathrm{E}$

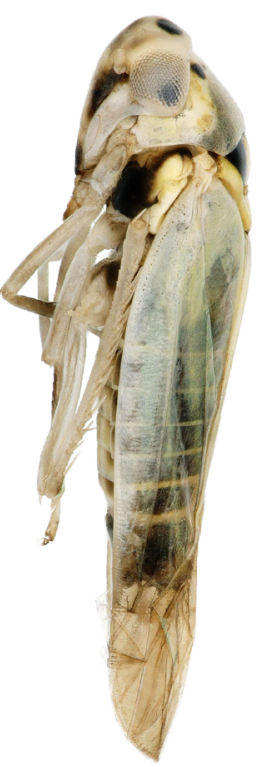

B

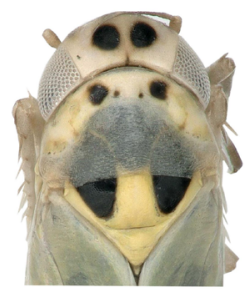

$\mathrm{D}$
$\mathrm{C}$

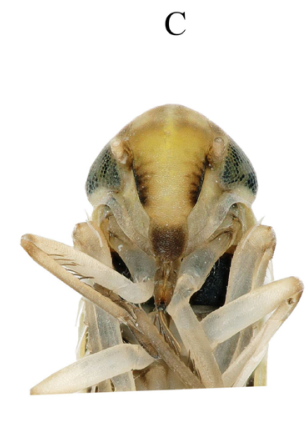

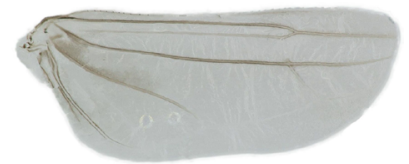

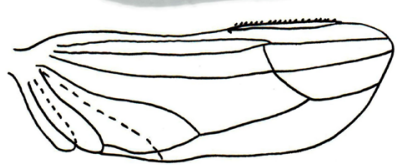

$\mathrm{F}$

Figure 1 Anuihuajianga pyramidalis Zhang \& Song gen. et sp. nov. A) Dorsal habitus B) Lateral habitus C) Head and thorax, dorsal view D) Face E) Forewing F) Hind wing.

base, $2^{\text {nd }}$ apical cell subquadrate, $3^{\text {rd }}$ apical cell widened distally, $4^{\text {th }}$ apical cell very small, triangular.

Abdominal apodemes small, extended to $3^{\text {rd }}$ sternite.

Male genitalia. Pygofer with lobe with posterodorsal margin bluntly angulate; with several macrosetae near middle of ventral margin; with distinct dorsal appendage movable, moderately long and tapered. Subgenital plate stubby; with narrow apex, abruptly subbase broadened; with macrosetae in two groups and peg-like setae on a preapical projection. Connective M-shaped; with central lobe broad, lateral arms long. Style with apical tooth longer than subapical one. Aedeagus without processes; shaft broadened, in ventral view; with enlarged dorsal apodeme in lateral view; gonopore at apex. Anal tube with segment $\mathrm{X}$ with paired basal processes and paired bifurcated ventroapical processes.

Distribution. China (Guizhou).

Etymology. The new genus name was formed by combining the prefix "Anu-" of the genus name "Anufrievia" and the type locality of its type species "Huajiang".

\section{Anuihuajianga pyramidalis Zhang \& Song sp. nov.}

urn:Isid:zoobank.org:act:BFF7904F-7D20-4B8E-A361-C737087595EB

(Figs. 1-3)

Diagnosis: see generic diagnosis.

Measurements. Male, 2.9-3.1 mm; female 3.2-3.3 mm (including wing).

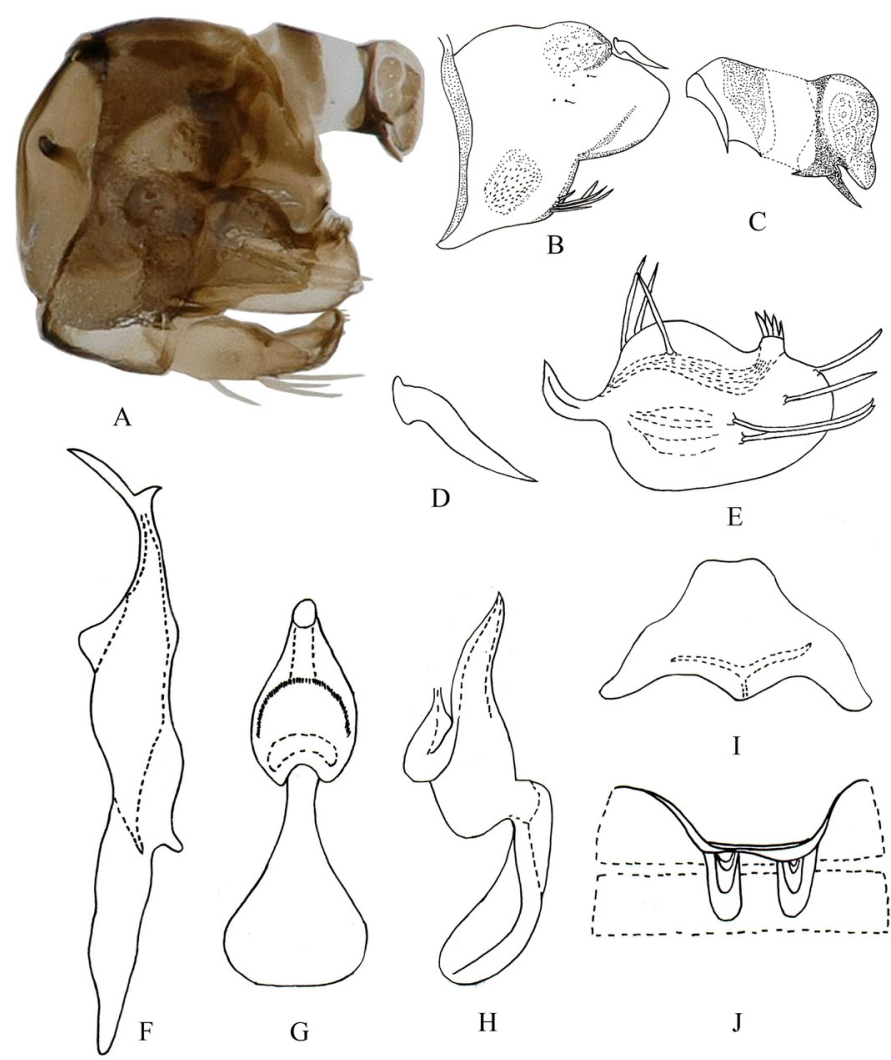

Figure 2 Anuihuajianga pyramidalis Zhang \& Song gen. et sp. nov., male A) Genital capsule, lateral view B) Pygofer lobe, lateral view C) Anal tube with appendages, lateral view D) Dorsal pygofer process, lateral view E) Subgenital plate, lateral view F) Style, ventral view $G$ ) Aedeagus, ventral view $\mathrm{H}$ ) Aedeagus, lateral view I) Connective, dorsal view J) Abdominal apodemes. 


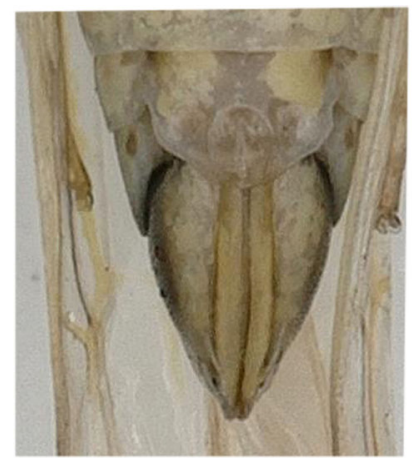

A

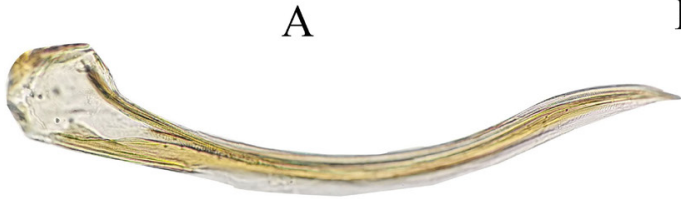

$\mathrm{C}$
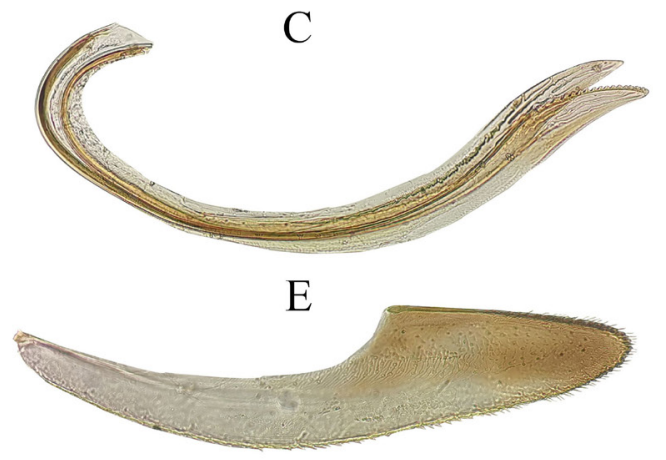

G

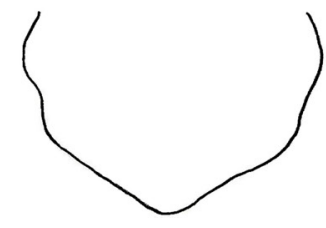

$\mathrm{B}$

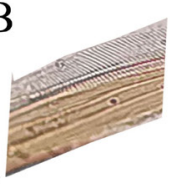

$\mathrm{D}$

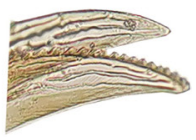

$\mathrm{F}$

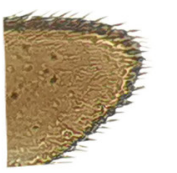

$\mathrm{H}$
Figure 3 Anuihuajianga pyramidalis Zhang \& Song gen. et sp. nov., female A) Terminalia, ventral view B) Sternite VII, ventral view C) Valvula I, lateral view D) Close up of dorsal sculpturing of valvula I E) Valvulae II, lateral view F) Close up of apex of valvulae II G) Valvula III, lateral view H) Close up of apex of valvula III.

Coloration. Vertex milky yellow, with pair of dark spots preapically; coronal suture short (Fig. 1A). Eyes greyish black (Fig. 1A). Face with frontoclypeus with both sides black, middle part yellow, anteclypeus black (Figs 1B, 1D). Pronotum grey; with pair of big black spots near anterior margin and pair of small black spots at inner sides of big black spots (Figs. 1A, 1C). Scutellum yellow, basal triangles black, transverse impression obvious (Fig. 1C). Forewing and hind wing semitransparent (Figs. 1E, 1F).

Male terminalia. Abdominal apodemes small, extended to hind margin of $3^{\text {rd }}$ sternite (Fig. 2J). Pygofer lobe with dorsal appendage falcate (Figs. 2A, 2B, 2D). Anal tube segment $X$ with paired ventroapical process (Figs. 2A, 2C). Subgenital plate with four macrosetae on outer surface; with region near base distinctly expanded and three moderately long macrosetae on dorsal margin; and four preapical peg-like setae on dorsal surface (Figs. 2A, 2E). Connective M-shaped (Fig. 2I). Aedeagus with shaft short; preatrium long (Figs. 2G, 2H). Style with two apical points (Fig. 2F).

Female genitalia. Seventh sternite broad, in ventral view (Figs. 3A, 3B). Valvula I elongate; curved dorsally and evenly tapered from base to apex; finely strigate along dorsal margin of apex (Figs. 3C, 3D). Valvula II asymmetrical; tooth-like protrusions on right blade (Figs. 3E, 3F). Valvula III tapered distally; apex rounded, with bushy microtrichae at apex (Figs. 3G, 3H).
Material examined. Holotype: $\lesssim$ CHINA, Guizhou Prov., Huajiang, 21 IX 2019, coll. Zhouwei Yuan and Xiao Yang. Paratypes: $10 \hat{\jmath} \widehat{\partial}, 17$ 우, same data as holotype.

Etymology. The name of the new species is a reference to the Latin word "pyramidalis" for the aedeagus shaped as overlapping cones.

\section{Discussion}

The new genus is similar to Anufrievia Dworakowska, 1970 in body shape, stubby subgenital plate, pygofer with group of macrosetae at ventrobasal angle and posterior margin truncate. However, it can be distinguished from the latter by the following characteristics: anal tube with paired basal and apical appendages; aedeagal shaft short, slightly "S" shaped, without atrial process; and connective with central lobe. This new genus is also somewhat similar to Arboridia Zachvatkin, 1946 in body shape and color pattern, especially with paired dark preapical spots on vertex, but differs in having the connective central lobe large, both dorsal pygofer and anal tube appendages present, and subgenital plate extremely short with a group of macrosetae at ventrobasal angle.

\section{Acknowledgments}

This work was supported by the World Top Discipline Program of Guizhou Province: Karst Ecoenvironment Sciences (No.125 2019 Qianjiao Keyan Fa); Guizhou Provincial Science and Technology Foundation ([2018]1411); Guizhou Science and Technology Support Project ([2019]2855); Science and Technology Project of Guiyang City ([2020]7-18); Innovation Group Project of Education Department of Guizhou Province ([2021]013); Training Program for High-level Innovative Talents of Guizhou Province ([2016]4020); and Project for Regional Top Discipline Construction of Guizhou Province: Ecology in Guiyang University [Qian Jiao Keyan Fa [2017]85].

\section{Conflicts of interest}

The authors declare no conflicts of interest.

\section{Author contribution statement}

NZ carried out the field work, including the collection of specimens, YHS carried out the taxonomic work, dissections, QFS carried out the preparation of photographs and plates, NZ wrote the initial manuscript, YHS participated in the corrections and final redaction.

\section{References}

Chen, X. X., Tan, C., Yuan, X. W., Song, Y. H., 2020. Cluster analysis of geographical distribution patterns of Erythroneurini in China. J. Environ. Entomol. 42 (5), 1141-1153.

Dietrich, C. H., 2005. Keys to the families of Cicadomorpha and subfamilies and tribes of Cicadellidae (Hemiptera: auchenorrhyncha). Fla. Entomol. 88, 502-517. https://doi.org/10.1007/0-387-31311-7_34.

Dmitriev, D. A., 2003. 3I Interactive Keys and Taxonomic Databases. Available in: http://dmitriev.speciesfile.org/ (accessed 25 June 2021).

Dworakowska, I., 1970. On the genera Asianidia Zachv. and Singapora Mahm. with the description of two new genera (Auchenorrhyncha, Cicadellidae, Typhlocybinae). Bull. Acad. Pol. Sci. biol. 18, 759-765. 
Guo, H. F., 2011. Major tea pests-advances in research on pseudo-eye leafhopper. Jiangsu Agric. Sci. 1, 132-134. https://doi.org/10.15889/j. issn.1002-1302.2011.01.09.

Jarrell, K. R., Rebek, E. J., Wayadande, A. C., Giles, K. L., 2020. Biology, ecology, and management of eastern grape leafhopper (Hemiptera: Cicadellidae), a key pest of vineyards in north America. J. Integr. Pest Manag. 11, 1-9. https://doi.org/10.1093/jipm/pmaa003.

Jiang, H., Saiki, T., Watanabe, K., Kwakita, H., Sato, M., 2005. Possible vector insect of mulberry dwarf phytoplasma, Tautoneura mori Matsumura. J. Gen. Plant Pathol. 71, 370-372. https://doi.org//10.1007/ s10327-005-0212-5.

Kim, H. G., Kabir, M. F., An, H. J., Mwamula, A. O., Oh, S., Jung, S. H., Lee, D. W., 2021. Seasonal phenology and damage by Singapora shinshana (Hemiptera: Cicadellidae) and other leafhoppers on ornamental trees in Korea. Entomol. Res. 51, 423-431. https://doi. org/10.1111/1748-5967.12520.

Morris, M. G., 1971. Differences between the invertebrate faunas of grazed and ungrazed chalk grassland, IV. Abundance and diversity of Homoptera-Auchenorrhyncha. J. Appl. Entomol. 8, 37-52. https:// doi.org/10.2307/2402126.
Roddee, J., Kobori, Y., Hanboonsong, Y., 2018. Multiplication and distribution of sugarcane white leaf phytoplasma transmitted by the leafhopper, Matsumuratettix hiroglyphicus (Matsumura) (Hemiptera: Cicadellidae), in infected sugarcane. Sugar Tech. 20, 445-453. https://doi.org/10.1007/s12355-017-0559-X.

Song, Y. H., Li Z. Z., 2013. Two new species of Empoascanara Distant (Hemiptera: Cicadellidae: Typhlocybinae) from Yunnan Province, China. Zootaxa 3637, 089-093. https://doi.org/10.11646/zootaxa.3637.1.11.

Song, Y. H., Li, Z. Z., Dai, R. H., 2016. A remarkable new genus and species of Erythroneurini (Hemiptera: Cicadellidae: Typhlocybinae) from China. Fla. Entomol. 99, 371-375. https://doi.org/10.1653/024.099.0305.

Song, Y. H., Li, C., 2017. A new erythroneurine leafhopper genus and species from Thailand (Hemiptera: Cicadellidae: Typhlocybinae). Zootaxa 4319, 379-382. https://doi.org/10.11646/zootaxa.4319.2.9.

Young, D. A., 1952. A reclassification of Western Hemisphere Typhlocybinae (Homoptera, Cicadellidae). Univ. Kans. Sci. Bull. 35, 3-217. https:// doi.org/10.5962/bhl.part.4327.

Zachvatkin, A. A., 1946. Studies on the Homoptera of Turkey. I-VII. Lond. Ent. Soc. Trans. 97, 148-176. https://doi.org/10.1111/j.1365-2311.1946. tb00278.x. 